\title{
Collapsing glomerulopathy following COVID-19 infection; possible relationship with APOL1 kidney risk alleles in African-Americans
}

\author{
Muhammed Mubarak ${ }^{1}$, Ramin Tolouian ${ }^{2}{ }^{(\mathbb{D}}$, Aiyoub Pezeshgi $^{3^{*}}$ \\ 'Department of Histopathology, SIUT, Karachi, Pakistan \\ ${ }^{2}$ Division of Nephrology, College of Medicine, University of Arizona, Tucson, AZ, USA \\ ${ }^{3}$ Department of Internal Medicine and Zanjan Metabolic Disease Research Center, School of Medicine, Zanjan University of Medical \\ Sciences, Zanjan, Iran
}

*Correspondence to

Aiyoub Pezeshgi,

Email;

Dr.a.pezeshki@gmail.com

Received 20 Apr. 2020

Accepted 28 Apr. 2020

Published online 5 May 2020

\section{Key point}

Collapsing glomerulopathy (CG) is being increasingly reported in African American patients with COVID-19 infection during the current pandemic. It is possible that CG following COVID-19 infection in this population may be linked to underlying APOL1 kidney risk alleles, which are not uncommon in this ethnic group. This lesion should be considered in the differential diagnosis of rapidly declining renal function in association with heavy proteinuria in the setting of COVID-19 disease, especially in patients of African ancestry.

Keywords: COVID-19, Acute kidney injury, Collapsing glomerulopathy, Chronic kidney disease, Cytokine storm syndrome, APOL1 kidney risk alleles

Citation: Mubarak M,
Tolouian R, Pezeshgi
A. Collapsing
glomerulopathy
following COVID-19
infection; possible
relationship with APOL1
kidney risk alleles in
African-Americans.
Immunopathol Persa.
2020;6(2):e18.
DOI:10.34172/
ipp.2020.18.

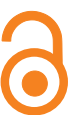

$\mathrm{D}$ ata has started accumulating on the extent and nature of renal involvement and its clinical manifestations in novel coronavirus disease 2019 (COVID-19), which is a disease primarily affecting the respiratory tract. The kidney involvement in COVID-19 disease is not uncommon, but the results of clinical studies are conflicting and very few biopsybased studies are available $(1,2)$. Most studies have suggested the tubulointerstitial compartment of kidney as the predominant site of injury, but more recently, a number of case reports, mostly from the United States, have interestingly detected collapsing glomerulopathy (CG) in the setting of COVID-19 infection (3-5). Larsen et al, described a 44-year-old African-American female, presenting with fever and pulmonary symptoms, who tested positive for SARS$\mathrm{CoV}-2$, by reverse transcriptase-polymerase chain reaction (RT-PCR) assay. During hospitalization, the patient developed an acute kidney injury (AKI), superimposed on previous chronic kidney disease (CKD), most probably attributable to her poorly controlled diabetes and nephroarteriosclerosis. The authors performed a kidney biopsy, which showed tuft collapse with overlying podocyte hyperplasia and hypertrophy in Bowman's space in some of the non-sclerotic glomeruli, and labelled it as CG (3). There was also a significant involvement of tubulointerstitial compartment of both acute and chronic nature. In addition, both the glomeruli and the tubulointerstitial compartments showed chronic changes of moderate degree in the background, a tell-tale sign of previous history of CKD. In fact, 14 out of 24 glomeruli were globally sclerosed. Notably, the patient was homozygous for high risk G1 allele on APOL1 genotyping of kidney tissue. This was the first renal biopsy-based report on renal pathology in COVID-19 disease, which raised the possibility that APOL1 high-risk alleles may augment the risk of renal injury in patients with COVID-19 infection (3).

Another case of CG in a 63-year-old black male patient with COVID-19 disease was described by Kissling et al (4). Soon after admission to the hospital, the patient developed AKI associated with heavy proteinuria. Renal biopsy was performed and revealed severe CG accompanied by pauci-inflammatory acute tubular necrosis (ATN). A RT-PCR for SARS-CoV-2 in RNA extracted from frozen biopsy piece was negative. However, electron microscopy

Copyright $\odot 2020$ The Author(s); Published by Nickan Research Institute. This is an open-access article distributed under the terms of the Creative Commons Attribution License (http://creativecommons.org/licenses/by/4.0), which permits unrestricted use, distribution, and reproduction in any medium, provided the original work is properly cited. 
revealed numerous spherical particles in vacuoles in podocytes cytoplasm, likely representing SARS-CoV-2. The results of these two tests have conflicting findings. The authors contended that the quality of extracted RNA was not optimal and RT-PCR technique is notorious for low sensitivity in non-respiratory samples. The most distinctive feature of COVID-19 involvement of the kidney in this case was the lack of significant inflammatory background as compared with lung involvement in this viral illness. The authors concluded that both CG and ATN have most probably resulted from direct viral toxicity rather than cytokine-associated damage. Kissling et al (4), however, did not perform APOL1 genotyping and the potential contribution of this risk factor to the development of CG could not be excluded in this case.

Peleg et al reported a case of CG in a 46-year-old West African man with COVID-19 infection (5). This patient was homozygous for G1 allele of APOL1 gene. Notably, pulmonary involvement was mild in this case, but AKI was severe and required renal replacement therapy. In-situ hybridization for SARS-CoV-2 performed on formalin-fixed paraffin embedded kidney tissue sections was negative for the receptor binding domain of the spike protein of the virus.

Interestingly, two of the three cases of CG in association with COVID-19 disease have been reported from the USA. CG has not been reported in this viral illness in China or Asia, probably because APOL1 high risk genotypes are only present in populations of African ancestry.

The kidney involvement in COVID-19 disease is not uncommon, but the results of clinical studies have shown divergent findings and very few biopsy-based studies are available. Most notably, Wang et al in an investigation on 116 hospitalized patients from Wuhan, China, concluded that infection by SARS-CoV-2 does not lead to acute renal impairment or aggravate CKD in COVID-19 patients (1). However, other reports have detected various manifestations of renal involvement during coronavirus disease is accompanied by in-hospital mortality (2). Additionally, the viral RNA and products in kidney tissue and urine of symptomatic patients of COVID-19 infection by a variety of techniques has been demonstrated (6). $\mathrm{Su}$ et al found viral particles in podocytes and tubular epithelial cells on ultrastructural study of postmortem specimens from kidneys of patients that died from COVID-19 disease (7). A high expression of angiotensinconverting enzyme 2 (ACE2) in the kidney, particularly in podocytes and proximal tubular epithelial cells, is a possible link to the susceptibility of renal parenchymal cells and direct viral invasion of kidney parenchyma following COVID-19 infection $(8,9)$. Another potential and more favored mechanism of COVID-19 mediated kidney injury is sepsis or systemic hyper-inflammation induced by cytokine storm syndrome provoked. The release of pro-inflammatory cytokines, inflammatory chemokines/mediators, and macrophage activation, indirectly mediate renal injury $(9,10)$. The predominant pattern of injury seems to be acute tubular injury with little or no involvement of glomeruli $(9,10)$. However, renal biopsies reported by Larsen et al (3), Kissling et al (4) and Peleg et al (5) showed both glomerular and tubulointerstitial involvement in patients of COVID-19. No viral RNA or virus-associated nucleoprotein in kidney tissues were detected in these cases, but Peleg et al did find SARS-CoV-2 like viral particles on electron microscopy of the biopsy. These findings lessen but not absolutely rule out the role of direct viral invasion as the cause of glomerular injury. African-American race is the common denominator of these three cases. This raises the role of race and genetic predisposition for developing CG in this population. Given the lack of direct demonstration of viral products in most of the cases, it is possible that genetically precarious podocytes and tubular epithelial cells are not able to withstand the onslaught of virus-induced cytokine storm syndrome and respond in the form of CG and severe ATN. It is also possible that the virus transiently invaded the kidney tissue and disappeared to undetectable limits after causing the lesions in the kidney. The glomerular lesions in patients with COVID-19 infection is similar to glomerular lesions that is more prevalent in AfricanAmerican population (11). It is possible that, CG following COVID-19 infection in African American patients, might be related to the APOL1 kidney high risk alleles which have been reported in $10-15 \%$ of this ethnic population (5). It is wise to keep CG in the differential diagnosis of any African-American patients who diagnose with COVID-19 infection and presents with significant proteinuria and rapid worsening of renal function $(5,11)$. A systematic review and meta-analysis of limited published data has shown that severe AKI in patients with COVID-19 disease is an ominous clinical predictor and is associated with high mortality (12).

In summary, CG is increasingly being reported in patients with COVID-19 disease, especially, those African origin. Presence of high risk alleles of APOL1 possibly increase the risk of development of CG in this ethnic group. These cases also raise the possibility of increased risk of developing kidney disease in such individuals in the setting of COVID-19 infection. There is a need to conduct more studies to elucidate the relationship between CG, APOL1 risk alleles and COVID-19 renal nephropathy.

Authors' contribution

Primary draft was prepared by AP and MM. RT and MM edited the paper. All authors read and signed the final paper.

Conflicts of interest

The authors report no conflict of interests.

Ethical considerations

Ethical issues (including plagiarism, data fabrication, double publication) have been completely observed by the authors.

Funding/Support

There was not financial support. 


\section{References}

1. Wang L, Li X, Chen H, Yan S, Li D, Li Y, et al. Coronavirus disease 19 infection does not result in acute kidney injury: an analysis of 116 hospitalized patients from Wuhan, China. Am J Nephrol. 2020. doi:10.1159/000507471

2. Cheng Y, Luo R, Wang K, Zhang M, Wang Z, Dong L, et al. Kidney impairment is associated with in-hospital death of COVID-19 patients. Kidney Int. 2020;97:829-838. doi: 10.1016/j.kint.2020.03.005.

3. Larsen C, Bourne T, Wilson J, Saqqa O, Sharshir M, Collapsing Glomerulopathy in a Patient with Coronavirus Disease 2019 (COVID-19). Kidney Int Rep. 2020. doi: 10.1016/j. ekir.2020.04.002.

4. Kissling S, Rotman S, Gerber C, Halfon M, Lamoth F, Comte D, et al. Collapsing glomerulopathy in a COVID-19 patient., Kidney Int. 2020. doi: 10.1016/j.kint.2020.04.006.

5. Peleg Y, Kudose S, D'Agati V, Siddall E, Ahmad S, Kisselev S, et al. Acute kidney injury due to collapsing glomerulopathy following COVID-19 infection. Kidney Int Rep. 2020. doi: 10.1016/j.ekir.2020.04.017.

6. Peng L, Liu J, Xu W, Luo Q, Deng K, Lin B, et al. 2019 novel coronavirus can be detected in urine, blood, anal swabs and oropharyngeal swabs samples. medrxiv. 21 February 2020. doi: 10.1101/2020.02.21.20026179.

7. Su H, Yang M, Wan C, Yi LX, Tang F, Zhu HY, et al. Renal histopathological analysis of 26 postmortem findings of patients with COVID-19 in China. Kidney Int. 2020. doi: 10.1016/j.kint.2020.04.003.

8. Li W, Moore MJ, Vasilieva N, Sui J, Wong SK, Berne MA, et al. Angiotensin-converting enzyme 2 is a functional receptor for the SARS coronavirus. Nature. 2003;426:450-4.

9. Li Z, Wu M, Guo J, Yao J, Liao X, Song S, et al. Caution on kidney dysfunctions of 2019-nCoV patients. medRxiv 2020. doi: 10.1101/2020.02.08.20021212.

10. Chu KH, Tsang WK, Tang CS, Lam MF, Lai FM, To KF, et al. Acute renal impairment in coronavirus-associated severe acute respiratory syndrome. Kidney Int. 2005;67:698-705.

11. Kopp JB, Nelson GW, Sampath K, Johnson RC, Genovese $\mathrm{G}$, An P, et al. APOL1 genetic variants in focal segmental glomerulosclerosis and HIV-associated nephropathy. J Am Soc Nephrol. 2011;22:2129-37. doi: 10.1681/ASN.2011040388.

12. Ali H, Daoud A, Mohamed MM, Salim SA, Yessayan L, Baharani J, et al. Survival rate in acute kidney injury superimposed COVID-19 patients: a systematic review and meta-analysis. Ren Fail. 2020;42:393-397. doi: 10.1080/0886022X.2020.1756323 